\section{Fade depth scaling with channel bandwidth}

W. Q. Malik, B. Allen and D. J. Edwards

The dependence of small-scale fading on bandwidth is quantified experimentally in the $3.1-10.6 \mathrm{GHz}$ band for indoor channels. The fade depth converges to $4 \mathrm{~dB}$ at $1 \mathrm{GHz}$ bandwidth, with little reduction for further increase in bandwidth. A simple yet accurate empirical fade depth model is developed, enabling convenient evaluation of the link budget for a channel with given bandwidth.

Introduction: Multipath propagation in wireless channels causes random fluctuations in the signal levels, leading to small-scale fading and system outage [1]. Recent research has analysed the impact of channel bandwidth on fading using information theoretic and statistical approaches. For example, an increase in the stochastic degrees of freedom with bandwidth in frequency-selective channels has been reported [2, 3]. This Letter investigates the variation of channel energy with bandwidth up to $7.5 \mathrm{GHz}$, spanning the FCC-allocated ultra-wideband (UWB) spectral range [4], and proposes a simple model to characterise this variation. Fade depth is a measure of the variation in the channel energy about its local mean due to small-scale fading. This determines the system fade margin, a key parameter used in link level design. The variation of fade depth with up to $10 \mathrm{MHz}$ bandwidth was analysed for code-division multiple-access (CDMA) channels in [5]. To our knowledge, there is no model describing the relationship of fade depth and bandwidth over a large bandwidth range, which would be of value to wideband and UWB system design.

Analysis methodology: For the analysis in this Letter, the small-scale spatial channel is measured by frequency-domain channel sounding with a vector network analyser to obtain the corresponding ensemble of complex channel transfer functions, denoted by $H(f)$. The measurements are performed at four different indoor locations representative of a typical small office environment. At each location, the receiving antenna is translated on a computer-controlled $30 \times 30$ element square grid with $3 \mathrm{~cm}$ inter-element separation. The $3.1-10.6 \mathrm{GHz}$ UWB band is measured in this manner under line-of-sight (LOS) and nonline-of-sight (NLOS) conditions to obtain 3600 UWB channel realisations. The frequency resolution of the measurement ensemble is 4.7 MHz, which is much smaller than the coherence bandwidth of the channel, evaluated as $30 \mathrm{MHz}$ [4]. Vertically polarised discone antennas, at $1.5 \mathrm{~m}$ height, are used for transmission and reception. For each data-set, the individual channel transfer functions are normalised with respect to the local mean path-loss, since large-scale propagation characteristics are not of interest here.

Consider the measured UWB channel, $H(f)$, with bandwidth $W=7.5$ $\mathrm{GHz}$ and centre frequency $f_{c}=6.85 \mathrm{GHz}$. On band partitioning using bandpass filters of bandwidth $W_{b}$ centred at $f_{c}$, the subbands of $H(f)$ are obtained, represented by $H_{b}(f), b=1, \ldots, N_{b}$, where $N_{b}$ is the number of such subbands. The channel energy can be calculated by the squared integration of the frequency-domain coefficients of $H(f)$ and $H_{b}(f)$ to obtain $E$ and $E_{b}$, respectively, i.e. $E_{b}=\int_{f_{l}}\left|H_{b}(f)\right|^{2}$ where, $f_{l}=f_{c}-W_{b} / 2$ and $f_{h}=f_{c}+W_{b} / 2$, and similarly for $E$. In our investigation we consider a range of values of $W_{b}$ such that it varies from an arbitrarily small value (a pure sinusoid) to the full-band $(7.5 \mathrm{GHz}$ wide) channel.

Fade analysis: The bandwidth, $W_{b}$, has a strong impact on the local variation of the $E_{b}$, as is observed from Fig. 1. It is apparent that the narrowband channel is far more susceptible to multipath interference and small-scale fading than the UWB channel. This is quantified by considering the standard deviation of this channel for the two bandwidths, which is found to be 4.6 and $0.5 \mathrm{~dB}$ for the narrowband and ultra-wideband cases, respectively. Furthermore, the cumulative distribution functions (CDF's) of $E_{b}$ are estimated for various values of $W_{b}$, as shown in Fig. 2. From the Figure, a $1 \mathrm{~Hz}$ channel will, on average, suffer fades deeper than $5 \mathrm{~dB}$ for $26 \%$ of the realisations, $7 \%$ for the $100 \mathrm{MHz}$ channel, $0.07 \%$ for the $500 \mathrm{MHz}$ channel, and practically $0 \%$ for the $7.5 \mathrm{GHz}$ channel. The progressive convergence of the channel energy to its mean, as a result of an increase in bandwidth, is obvious and a nonlinear relationship between $W_{b}$ and the variance can be observed. This behaviour is next investigated comprehensively and the empirical relationship between channel bandwidth and fading is derived.

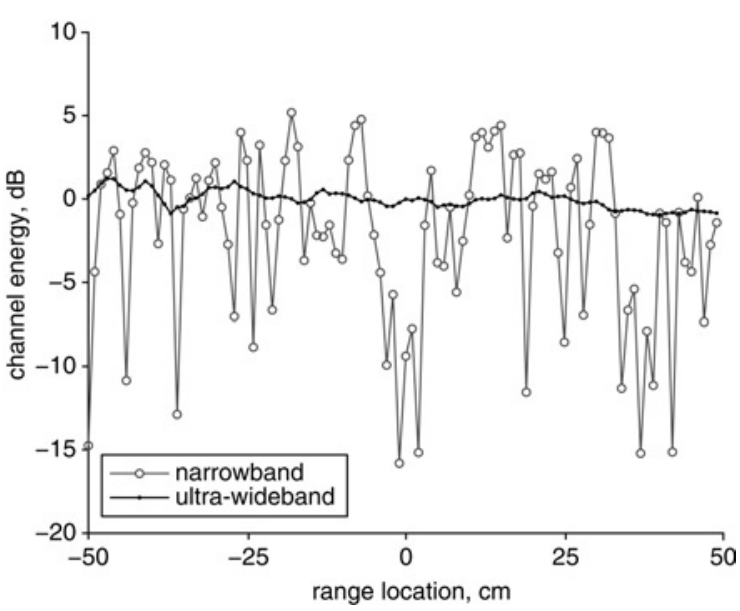

Fig. 1 Example of small-scale fading illustrated by spatial variation of channel energy, normalised to local mean energy, for narrowband $(1 \mathrm{~Hz})$ and ultra-wideband channels $(7.5 \mathrm{GHz})$ in indoor LOS environment

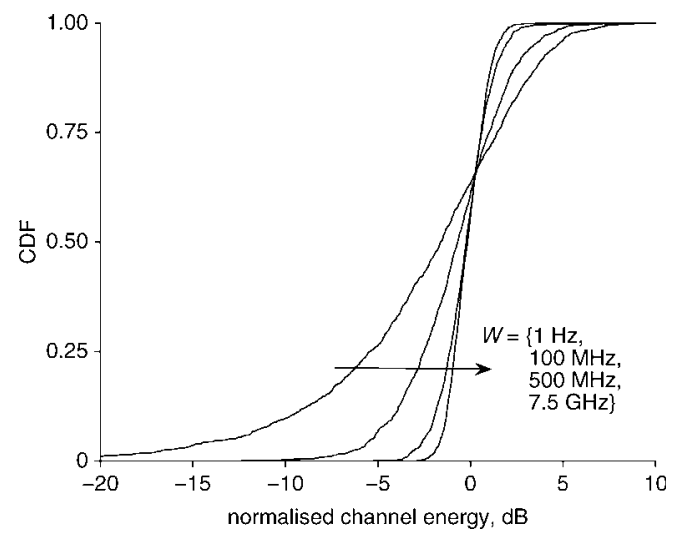

Fig. 2 Cumulative distribution functions (CDF's) of channel energy, normalised to local mean energy, for various values of channel bandwidth, $W_{b}$

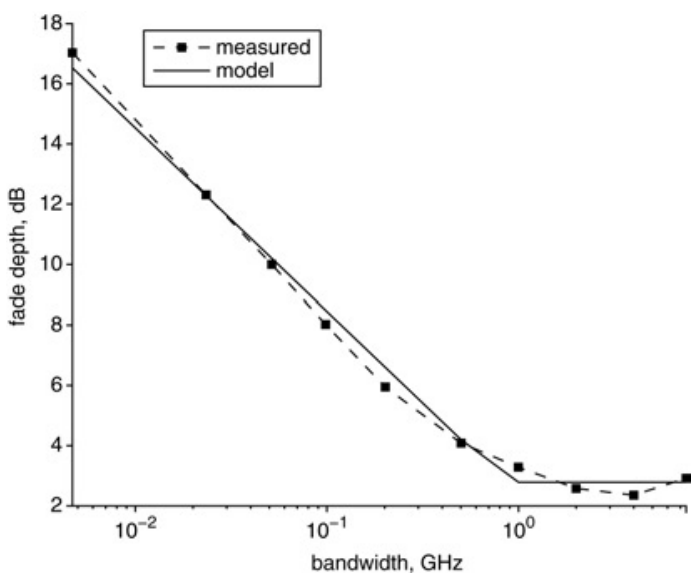

Fig. 3 Dual-slope model for prediction of fade depth, $F_{b}$ (measured LOS fade depth shown for comparison)

Fade depth modelling: The fade depth, $F$, is the observed range of local spatial variation in channel energy. It can be evaluated in terms of standard deviation, $\sigma$, of the channel energy expressed in dB scale. We define $F$ to span the $3 \sigma$ range of $E_{b}$, so the number of standard deviations considered, $s$, is 3 . The fade depth, $F_{b}$, is then computed corresponding to a number of values of $W_{b}$, from the measurement data. Fig. 3 shows the value of $F_{b}$ averaged across the channel ensemble, expressed as a function of $W_{b}$. From our data, the $3 \sigma F_{b}$ is found to be about $17 \mathrm{~dB}$ for a narrowband LOS channel, falling to about $4 \mathrm{~dB}$ at $W_{b}=1 \mathrm{GHz}$, after which it is nearly constant. A linear relationship can be approximated between $F_{b}$ and $\log _{10} W_{b}$, when $W_{b}=[0,1] \mathrm{GHz}$, beyond which $F_{b}$ is almost constant. This result suggests the applicability of a dual-slope linear regression fit with a breakpoint at the critical bandwidth, $W_{0}=1$ $\mathrm{GHz}$. A higher-order polynomial may provide a better approximation but 
a dual-slope model is adopted here for its simplicity and intuitiveness. Thus we estimate $F_{b}$ by

$$
\hat{F}_{b}= \begin{cases}s\left(k_{1}-k_{2} \log _{10} W_{b}\right), & \text { if } W_{b}<W_{0} \\ s k_{3}, & \text { otherwise }\end{cases}
$$

where $W_{0}$ and $k_{i}, i=\{1,2,3\}$ are the model parameters. For the linear region $W_{b}<W_{0}$, the model parameters are obtained as the intercept and slope of the regression line defined by $\log _{10} W_{b}$ against $F_{b}$, so that we have $k_{1}=F_{b} /\left.s\right|_{W_{b} \rightarrow}$ and $k_{2}=\left(s k_{1}-F_{b}\right) / s \log _{10} W_{b}$. For the region $W_{b} \geq W_{0}$, we have the parameter $k_{3}=\varepsilon_{\mathcal{K}}\left\{F_{b}\right\}$, where $\mathcal{K}$ defines the set of channels for which $W_{b} \geq W_{0}$, and $\varepsilon_{\mathcal{K}}$ denotes expectation over $\mathcal{K}$. From empirical analysis, the best-fit values of $k_{i}, i=\{1,2,3\}$, are found to be 5.6, 1.7 and 1.4 in LOS channels, and 6.3, 2.0 and 0.9 in NLOS channels, respectively. With these parameters, the proposed model allows the prediction of $F_{b}$ for a given channel bandwidth, $W_{b}$. The proposed LOS model is plotted in Fig. 3 and shows a close fit to the measured data.

The LOS environment exhibits lower $k_{l}$ than NLOS, implying that its narrowband fading is less severe, as demonstrated by its $3 \sigma$ fade depth of $17 \mathrm{~dB}$, compared to $19 \mathrm{~dB}$ in NLOS. Similarly, the comparison of $k_{2}$ indicates that an increase in bandwidth provides somewhat less fade mitigation in LOS than in NLOS. Finally, the $3 \sigma$ fade depth at asymptotically large bandwidth, given by $s k_{3}$, is $3-4 \mathrm{~dB}$, which is consistent with previous UWB channel studies [4]. On the basis of the insignificant difference in the LOS and NLOS model parameters, the UWB fade depth can be considered to be relatively insensitive to the propagation environment as far as the presence of LOS is concerned. The reason is that a large number of multipath components can be individually resolved in a UWB channel, with the result that the tap corresponding to the LOS component carries a relative portion of the total channel energy [4].

The maximum magnitude of the model prediction error is $0.5 \mathrm{~dB}$, occurring at $W_{0}$, while the asymptotic error at large bandwidth is $0.1 \mathrm{~dB}$. Thus the proposed model provides a close approximation to the measured fade depth. As the model is parametric in $s$, it can be easily extended to the analysis of fade depth defined in terms of any given number of standard deviations of channel energy, such as $s=1$ or 6 , which would correspond to the desired system performance and outage levels.

The empirical model presented in this Letter, applicable universally to narrowband, wideband and UWB channels, enables the wireless system designer to predict the impact of bandwidth on multipath fading. It allows the determination of the optimal operating bandwidth with the desired trade-offs between the quality of service, implementation complexity and resource utilisation.

Acknowledgment: The financial support of the UK Engineering and Physical Sciences Research Council via grant GR/T21769/01 is gratefully acknowledged.

(C) The Institution of Engineering and Technology 2007

30 July 2007

Electronics Letters online no: 20072222

doi: 10.1049/el:20072222

W. Q. Malik (Laboratory for Information and Decision Systems, Massachusetts Institute of Technology, 77 Massachussetts Avenue, Cambridge, MA 02139, USA)

E-mail: wqm@mit.edu

B. Allen and D. J. Edwards (Department of Engineering Science, University of Oxford, Parks Road, Oxford OX1 3PJ, United Kingdom)

\section{References}

1 Rappaport, T.S.: 'Wireless communications: principles and practice' (Prentice Hall, 2001, 2nd edn.)

2 Hayar, A.M., Knopp, R., and Saadane, R.: 'Subspace analysis of indoor UWB channels', EURASIP J. Appl. Signal Process., 2005 2005, (3), pp. 287-295

3 Schuster, U.G., Bolcskei, H., and Durisi, G.: 'Ultra-wideband channel modeling on the basis of information-theoretic criteria'. Proc. Int. Symp. Information Theory (ISIT), Adelaide, Australia, September, 2005

4 Allen, B., Dohler, M., Okon, E.E., Malik, W.Q., Brown, A.K., and Edwards D.J. (Ed.): 'Ultra-wideband antennas and propagation for communications, radar and imaging' (Wiley, London, UK, 2006)

5 Wilson, P., Papazian, P., Cotton, M., and Lo, Y.: 'A comparison of $1920 \mathrm{MHz}$ mobile channel diversity gain using horizontal and vertical arrays', IEEE Trans. Commun., 2001, 49, (12), pp. 2068-2070 